\title{
Sketch-based Modeling of Parameterized Objects
}

\author{
Chen Yang
}

Dana Sharon

Michiel van de Panne

\author{
University of British Columbia*
}

\section{Introduction}

Sketch-based modeling holds the promise of making 3D modeling accessible to a significantly wider audience than current modeling tools. We present a modeling system that is capable of constructing 3D models of particular object classes from 2D sketches. The core of the system is a sketch recognition algorithm that seeks to match the points and curves of a set of given 2D templates to the sketch. The matching process employs an optimization metric based on curve feature vectors, and the search space of possible correspondences is restricted by encoding knowledge about relative locations of object parts into the 2D template. Once a best-fit template is found, a $3 \mathrm{D}$ object is constructed using a series of measurements that are extracted from the labelled 2D sketch. We apply our sketchrecognition and modeling algorithms to sketches of cups and mugs, airplanes, and fish. The system allows non-experts to quickly create 3D models of specific object classes from sketches or by tracing over photographs.

Previous sketch-based methods such as Teddy[Igarashi et al. 1999] use a vocabulary of gestural pen strokes to incrementally develop the shape of the 3D object. In contrast, our sketch-based system works directly from a drawing of the final object, at the expense of requiring a priori domain knowledge.

\section{Sketch Recognition}

As a preprocess, each pen stroke is split into segments at points of local curvature maxima. A graph structure is then created from these stroke segments. Graph nodes are assigned to the start and end of each segment and nearby nodes are merged. Graph edges consist of the stroke segments.

Object classes are represented as a hierarchy of part templates. These templates are also graph structures and their purpose is to classify stroke segments in the sketch as specific object parts. The system supports a flexible instantiation of the part templates. Parts may be deemed as mandatory or optional. A choose-one-of-N option may also be specified. For example, a cup template can specify that it may have an optional right handle and that the system should choose between a rounded handle part template and a square handle template, depending on which one fits best. The ability to support this type of information makes our recognition approach different from other shape recognition approaches that support only a flat hierarchy, i.e., given $\mathrm{N}$ fixed templates, find the best-fit template.

Since both the sketch and part templates are represented as graphs, the actual process of matching them is accomplished using a search over graph node correspondences. A given node correspondence is scored using a curve-matching metric on the induced edge correspondences. Once strokes have been classified and labelled according to the hierarchy of part templates, the recognition process is complete. To deal with multiple object classes, we match against each class template in sequence and retain the best result.

A new class of objects can be easily added to the system by designing the hierarchy of part templates and developing a procedural means of constructing the 3D shape from the labelled sketch.

\section{3D Modeling}

$3 \mathrm{D}$ geometry is constructed for the sketch using a variety of techniques. These include scaled instancing of parts, such as the plane engines, using extrusions, for example to create a plane wing, or using a surface of revolution to create a cup body.

Parameters that can be extracted from a labelled sketch include lengths, such as for a plane wing, or the control points of a fitted spline, such as for the curve defining the shape of a plane fuselage or cup handle.

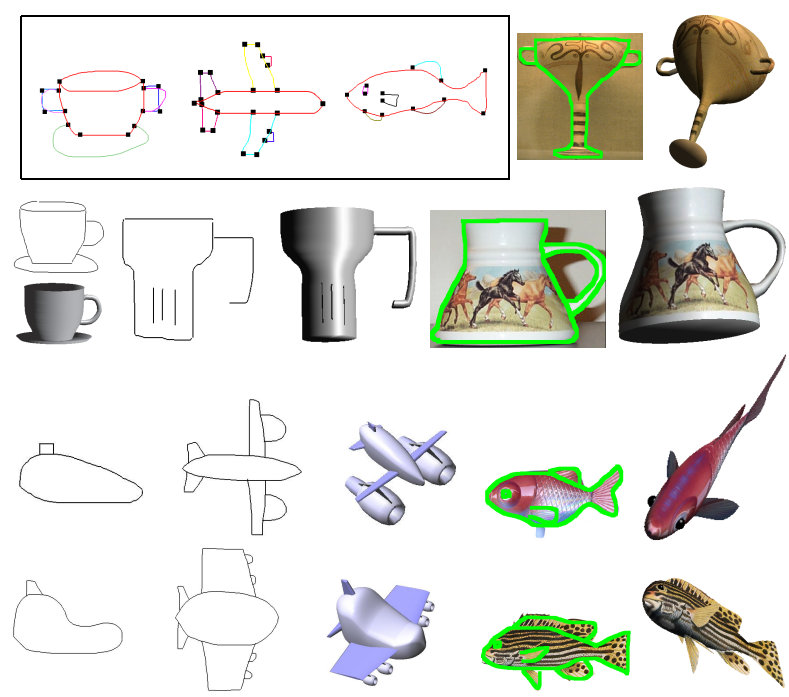

Figure 1: Templates (in box) and sketches of cups, planes and fish, shown together with the synthesized 3D models.

\section{Conclusion}

We have presented a system that supports quick-and-dirty creation of 3D models based upon a sketching interface. Instead of a gestural interpretation of pen strokes, a template-based sketch recognition algorithm has been proposed which treats strokes as a sparselypopulated image and then drives the instantiation of a flexiblyparameterized 3D model. Future work will focus on improving scalability and recognition robustness by automatically learning part template distributions using training data. Application areas of our system include user-driven content creation for games and domain-specific modeling programs.

\section{References}

Igarashi, T., Matsuoka, S., And TANaKa, H. 1999. Teddy: a sketching interface for $3 \mathrm{~d}$ freeform design. In SIGGRAPH '99, ACM Press/Addison-Wesley Publishing Co., 409-416. 